Nervenarzt 2021 · 92:1276-1282

https://doi.org/10.1007/s00115-021-01155-4

Angenommen: 10. Mai 2021

Online publiziert: 7. Juli 2021

(c) Der/die Autor(en) 2021

\section{Einführung}

Multiple Sklerose (MS) stellt die häufigste neurologische Erkrankung dar, die zu einer Behinderung im jungen Erwachsenenalter führt und betrifft mehr als 2,3 Mio. Menschen weltweit [9]. Infektionen bergen generell ein Risiko für MSPatienten, da sie nicht nur einen Pseudoschub auslösen können, sondern auch mit Krankheitsprogression und reduzierter Lebenserwartung assoziiert sind [40, 46, 82]. Dieses infektionsassoziierte Risiko kann sich durch entsprechende Begleiterkrankungen und krankheitsmodifizierende Therapien (DMTs) nochmals weiter erhöhen [23, 39, 54, 62, 81]. Darüber hinaus existieren verschiedene Theorien zur Beteiligung von Infektionen an der Immunpathogenese der MS. Diese umfassen u.a. folgende Mechanismen: „molecular mimicry“, „bystander activation“" und „epitope spreading“ [44].

Mit dem Ausbruch der COVID-19Pandemie Ende 2019 in der Provinz Hubei in China stehen daher insbesondere Menschen mit MS vor neuen Herausfor-

Teil 2 dieses Beitrags „Die Corona-Pandemie und Multiple Sklerose: Impfungen und deren Implikationen für Patienten - Teil 2: Impfstofftechnologien" finden Sie unter https://doi.org/ 10.1007/s00115-021-01154-5.

Tobias Monschein · Tobias Zrzavy' · Micha Löbermann ${ }^{2}$ Alexander Winkelmann ${ }^{3}$. Thomas Berger ${ }^{1} \cdot$ Paulus Rommer ${ }^{1,5} \cdot$ Hans-Peter Hartung ${ }^{1,4} \cdot$ Uwe K. Zettl $^{5}$

${ }^{1}$ Universitätsklinik für Neurologie, Medizinische Universität Wien, Wien, Österreich

${ }^{2}$ Abteilung für Tropenmedizin und Infektionskrankheiten, Universitätsmedizin Rostock, Rostock, Deutschland

${ }^{3}$ Klinik und Poliklinik für Neurologie, Universitätsmedizin Rostock, Rostock, Deutschland

${ }^{4}$ Klinik für Neurologie, Universitätsklinikum Düsseldorf, Medizinische Fakultät, Heinrich-Heine-Universität, Düsseldorf, Deutschland

${ }^{5}$ Klinik und Poliklinik für Neurologie, Neuroimmunologische Sektion, Universitätsmedizin Rostock, Rostock, Deutschland

\title{
Die Corona-Pandemie und Multiple Sklerose: Impfungen und deren Implikationen für Patienten - Teil 1: Empfehlungen
}

derungen. Hier kommen nicht nur im Bereich der Patientenversorgung und Immuntherapien Fragen auf, sondern vor allem auch Fragen, die das Impfwesen betreffen [29, 57, 69]. Inzwischen wurden zahlreiche Impfstoffe weltweit zugelassen, darunter 2 mRNA- und 2 Vektorimpfstoffe in der Europäischen Union (EU; $[4,59,77,85])$. Angesichts der enormen Fortschritte im Bereich der Vakzinenherstellung auf der einen Seite und diverser Virusmutationen auf der anderen Seite ist ein hoher Informationsbedarf, sowohl für das Gesundheitspersonal als auch Menschen mit MS, gegeben. Ziel dieser Arbeit ist es daher, einen Überblick über COVID-19, Manifestationen als auch das Impfwesen und dessen technologische Fortschritte zu geben und deren Implikationen für Menschen mit MS herauszuarbeiten und entsprechende Empfehlungen zu generieren.

\section{COVID-19 Hintergrund}

COVID 19 ist eine Infektionskrankheit, die durch das hochansteckende RNAVirus SARS-CoV-2 („severe-acute-respiratory-syndrome corona-virus-2“) verursacht wird. Insgesamt wurden bisher 7 humanpathogene Coronaviren beschrieben, darunter auch das „severe acute respiratory syndrome corona-virus" (SARS-CoV, Ausbruch 2002) sowie das „middle east respiratory syndrome coronavirus" (MERS-CoV, Ausbruch 2012) mit ebenfalls zoonotischen Ursprüngen [13]. Des Weiteren konnte gezeigt werden, dass eine hohe genetische Übereinstimmung (bis zu 79\%) mit anderen Coronaviren gegeben ist [36]. Die Übertragung auf den Menschen ist bislang nicht geklärt, es gibt jedoch Evidenz aus phylogenetischen Studien, dass diese am ehesten von der Fledermaus und dem Pangolin ausgeht [19]. Bis zum 24.04.2021 wurden von Seiten der WHO 145.216.414 bestätigte SARS-CoV-2-Fälle sowie 3.079 .390 Todesfälle registriert [86]. Die aktuelle COVID-19-Pandemie stellt damit eine weltweite Gesundheitskrise dar. Eines der Hauptprobleme von COVID-19 ist die Übertragung von SARS-CoV-2 auch von asymptomatischen und präsymptomatischen Personen, im Gegensatz zu SARS-CoV [78, 79]. Der hauptsächliche Infektionsweg scheint via Aerosole/ Tröpfcheninfektion zu erfolgen, es werden jedoch auch andere Infektionswege wie via Kontakt oder kontaminierte Oberflächen diskutiert [87].

Des Weiteren bindet SARS-CoV-2, ähnlich wie SARS-CoV, an ,angiotensin- 
converting enzyme 2“ (ACE-2), um in respiratorische Epithelzellen einzudringen, weist jedoch eine 10 - bis 20-fach höhere Bindungsaffinität als SARS-CoV auf [33]. Nach dem Eindringen in die respiratorischen Epithelzellen beginnt SARS-CoV-2 mit der Replikation und raschen Ausbreitung, bis in die alveolären Epithelzellen der Lunge. Das angeborene Immunsystem bewerkstelligt eine erste unspezifische, sofortige Immunreaktion. Dabei wird das Spike-Protein von Erregererkennungsrezeptoren („pathogen-associated molecular patterns“, PAMPS) erkannt und führt über diverse Signalkaskaden zu einer Aktivierung von Immunzellen mit konsekutiver Freisetzung von Signalmolekülen, die wiederum eine Rekrutierung weiterer Immunzellen begünstigen z.B. durch eine gesteigerte Interferonproduktion. Dies wiederum verzögert die Virusausbreitung und führt in der Folge zur Aktivierung des adaptiven Immunsystems. Durch Präsentation spezifischer Abschnitte des Virus mittels Haupthistokompatibilitätskomplex I oder II antigenpräsentierender Zellen, wie z.B. Makrophagen, folgt die Einleitung einer virusspezifischen Immunantwort mit Aktivierung und klonaler Expansion von $\mathrm{CD}^{+}{ }^{+}$sowie $\mathrm{CD}^{+}{ }^{+} \mathrm{T}$-Zellen und die Generierung von Plasmazellen mit konsekutiver Produktion von Antikörpern [63]. Durch die T-Zell-Aktivierung, insbesondere $\mathrm{CD}^{+}$- T-Zellen, kommt es zu einer Elimination von bereits infizierten Zellen, während die CD4+-T-Zellen hier unterstützend durch das Instruieren von $\mathrm{B}-Z$ ellen, das Unterstützen von $\mathrm{CD}^{+}$T-Zellen, das Rekrutieren angeborener Immunzellen sowie eine direkte antivirale Aktivität wirken [63, 67, 70, 74]. Je nach Schwere der Infektion kann eine rapide Erregerausbreitung zusammen mit begleitender Gewebedestruktion ein Zytokin-Sturm-Syndrom (CSS) verursachen [16, 42]. Dieses kann in einem akuten Atemnotsyndrom (ARDS) resultieren, wofür wiederum Alter und Komorbiditäten die wichtigsten Risikofaktoren darstellen [58, 84].

Insbesondere für Patienten mit $\mathrm{Au}$ toimmunerkrankungen wie der MS und gegebenenfalls entsprechender immunmodulatorischer und/oder immunsup- pressiver Therapie ist es von Interesse, eine entsprechende Immunantwort nach durchgemachter SARS-CoV-2-Infektion und/oder Impfung nachzuweisen (unter der Voraussetzung verfügbarer Cut-offs für eine Immunprotektion). Für den Nachweis der B-Zell-Immunität steht beispielsweise der ELISA („enzyme-linked-immunoassay“) zur Detektion des Antikörpertiters zur Verfügung. Mittels zellbasierter Verfahren (Neutralisationstest) kann darüber hinaus der Anteil an neutralisierenden Antikörpern nachgewiesen werden. Ein Teil der Antiköper wirkt neutralisierend, andere erfüllen Funktionen wie Opsonierung oder Komplementaktivierung $[17,65]$. Für den Nachweis einer T-ZellImmunität können Verfahren wie der ELISpot („enzyme linked immuno spot assay") oder eine Durchflusszytometrie (z.B. „fluorescence-activated cell sorting" [FACS ]) angewendet werden [53]. In einer rezenten Studie von Achiron et al. wurde die humorale Immunantwort anhand des Antikörpertiters (ELISA) bei Gesunden, MS-Patienten mit und ohne Therapie verglichen und es konnte eine adäquate Immunantwort für alle, ausgenommen einem Teil der Patienten mit Ocrelizumab sowie den meisten Patienten mit Fingolimod als DMT, gezeigt werden [2].

\section{Neurologische Beteiligungen bei COVID-19}

Die Manifestationen von COVID-19 gestalten sich vielseitig. Die Symptome reichen von Fieber, trockenem Husten und Müdigkeit bis hin $\mathrm{zu}$ respiratorischem Versagen mit fatalem Ausgang [24, 73, 80]. Außerdem konnte gezeigt werden, dass ACE-2 nicht nur in der Lunge, sondern auch im Ösophagus, Ileum und Kolon exprimiert wird. Angesichts dessen sowie Durchfallraten von bis zu $50 \%$ stellt der Gastrointestinaltrakt ebenfalls eine mögliche Eintrittspforte für das Virus dar $[14,83]$. Neben diesen systemischen Manifestationen gibt es zunehmend Evidenz für eine Mitbeteiligung des Nervensystems. Dies kann von einer milden Hyposmie über parainfektiöse Syndrome bis hin zu Enzephalopathien und Schlaganfällen gehen $[18,56]$.
Neurologische Mitbeteiligungen finden sich darüber hinaus auch häufig bei hospitalisierten Kindern und Jugendlichen [31]. Eine mögliche Route ist die Migration in das ZNS mittels axonalen Transports über olfaktorische Neurone. Auffällig sind in diesem Zusammenhang auch die häufigen Hyposmien sowie MRT-Veränderungen im olfaktorischen Kortex [60]. Eine weitere Möglichkeit wäre das Überwinden der Blut-Hirn-Schranke (BHS), was via Internalisierung und/oder inflammationsgetriggerte Funktionsstörung der BHS erfolgen kann [25]. Eine dritte Möglichkeit stellen infizierte Immunzellen dar, die ins Gehirn einwandern und auch als Reservoir dienen können [6].

Überblicksmäßig ergeben sich 5 Hauptkategorien für neurologische Manifestationen im Zuge von COVID-19: (1) Enzephalopathie, (2) entzündliche ZNSSyndrome wie Enzephalitis oder ADEM, (3) ischämische Schlaganfälle, (4) periphere Beteiligungen wie das GuillainBarre-Syndrom (GBS) und (5) andere Manifestationen [56]. Bezüglich GBS und COVID-19 gibt es widersprüchliche Befunde. So zeigen einzelne epidemiologische Untersuchungen keine Assoziation von GBS mit COVID-19 [28]. Darüber hinaus wurde in einer rezenten Arbeit von Nalbandian et al. das postakute COVID-19-Syndrom (>4 Wochen nach Infektion) beschrieben, welches sich in bis zu $38 \%$ der Fälle in Form neurologischer Symptome äußert (Fatigue, Kopfschmerzen, Dysautonomie, kognitive Beeinträchtigung; [49]). Einen tabellarischen Überblick mit den entsprechenden einzelnen Diagnosen sowie vermuteten Pathomechanismen soll - Tab. 1 geben.

\section{Multiple Sklerose und die COVID-19-Pandemie}

Multiple-Sklerose-Patienten scheinen per se nicht einem erhöhten Risiko für eine SARS-CoV-2-Infektion oder einen schwereren Verlauf zu unterliegen [26, 55]. Daten aus Italien und Frankreich konnten, neben bekannten allgemeinen kardiovaskulären Risikofaktoren wie Adipositas, vor allem Alter und Behinderungsgrad (Expanded Disability Status 
Scale, EDSS) als Risikofaktoren für einen schwereren Verlauf von COVID-19 bei MS-Patienten herausarbeiten [35, 72]. Des Weiteren wurde in der italienischen Studie eine erhöhte Anzahl an schwereren COVID-19-Fällen unter MS-Patienten mit B-Zell-depletierenden Therapien detektiert. Im Gegensatz dazu konnte in der französischen Studie keine Assoziation zwischen DMTs und COVID19-Schwere aufgezeigt werden. Mögliche Risiken, die im Zusammenhang mit B-Zell-depletierenden Therapien diskutiert werden, sind eine beeinträchtigte antivirale erworbene Immunität (reduzierte Antikörpertiter sowie reduzierte T- und B-Zell-Interaktion), ein erhöhtes Reinfektionsrisiko, eine beeinträchtigte Impfantwort sowie die assoziierte mögliche Hypogammaglobulinämie.

Auf der anderen Seite gibt es diverse Komplikationen einer SARS-CoV-2Infektion, z. B. Hyperinflammation/CSS und dadurch in weiterer Folge das für einen fatalen Verlauf entscheidende ARDS, die durch eine solche Therapie positiv beeinflusst werden könnten [43].

In diesem Zusammenhang sind mögliche positive Einflüsse auf die COVID19-Schwere auch durch Interferon- $\beta$ (antivirale Wirkung) oder monoklonale Antikörper gegen den Interleukin-6-Rezeptor (Tocilizumab) beschrieben worden [21, 27, 64]. Ergänzend dazu zeigte eine Studie von Alonso et al. eine erhöhte Hospitalisierungsrate bei NMOSD-Patienten $(n=16)$ gegenüber MS-Patienten $(n=129)$. Des Weiteren starb in dieser Studie ein Drittel der NMOSD-Patienten, von denen alle unter einer Therapie mit Rituximab standen. Hier gilt es jedoch, die geringe Gesamtfallzahl an NMOSDPatienten $(n=16)$ zu berücksichtigen [3].

Unter Zusammenschau rezenter Evidenz bleibt jedoch weiterhin ungeklärt, inwiefern eine COVID-19-Infektion und deren Verlauf durch DMTs beeinflusst werden [30]. Angesichts der fortbestehenden COVID-19-Pandemie werden zukünftige umfangreichere Therapieregisterdaten von MS-Patienten mehr Klarheit über den Einfluss von DMTs bringen. Insgesamt scheinen jedoch Risikofaktoren wie Alter, Behinderungsgrad (EDSS) und Komorbiditäten für den Verlauf bzw. die Schwere einer SARS-

Nervenarzt 2021 · 92:1276-1282 https://doi.org/10.1007/s00115-021-01155-4

(c) Der/die Autor(en) 2021

\section{T. Monschein · T. Zrzavy · M. Löbermann · A. Winkelmann · T. Berger · P. Rommer · H.-P. Hartung · U. K. Zettl \\ Die Corona-Pandemie und Multiple Sklerose: Impfungen und deren Implikationen für Patienten - Teil 1: Empfehlungen}

Zusammenfassung

Die aktuelle COVID-19-Pandemie stellt eine weltweite Gesundheitskrise dar. Nicht zuletzt mit dem Aufkommen diverser Virusmutationen entstehen insbesondere für das Gesundheitswesen laufend neue Herausforderungen. Angesichts der Vielfalt an immunmodulatorischen und immunsuppressiven Therapien für die Multiple Sklerose (MS) sowie der enormen Entwicklungen im Bereich der Vakzinenherstellung besteht ein hoher Informationsbedarf für Menschen mit MS. Ziel dieser Arbeit ist es daher, einen Überblick über MS und COVID-19 sowie die damit einhergehenden Implikationen für MS-Patienten, insbesondere das Impfwesen betreffend, aufzuarbeiten und entsprechende Empfehlungen auszusprechen.

Schlüsselwörter

Multiple Sklerose · Impfen · SARS-CoV-2 . COVID-19. Pandemie

\section{The corona pandemic and multiple sclerosis: vaccinations and their implications for patients_-Part 1: recommendations}

\section{Abstract}

The ongoing COVID-19 pandemic is a global health crisis. New challenges are constantly emerging especially for the healthcare system, not least with the emergence of various viral mutations. Given the variety of immunomodulatory and immunosuppressive therapies for multiple sclerosis (MS) and the immense developments in vaccine production, there is a high need of information for people with MS. The aim of this article is therefore to provide an overview of MS and COVID-19 as well as to clarify the implications for patients with MS, especially regarding vaccination and to formulate appropriate recommendations.

Keywords Multiple sclerosis - Vaccination - SARS-CoV-2 . COVID-19. Pandemic
CoV-2-Infektion viel entscheidender zu sein, als DMTs [7, 10, 23, 38, 39, 41, 62]. Auf dem bisherigen Wissensstand aufbauend wird gemäß den jeweiligen regionalen Empfehlungen einheitlich die Fortführung immunmodulatorischer Therapien wie bisher empfohlen. Bei zyklischen Therapien wie Ocrelizumab, Rituximab, Alemtuzumab oder Cladribin kann jedoch je nach individueller Situation (Komorbiditäten, Alter, EDSS, Lymphozytenzahl, Krankheitsaktivität) und in Absprache mit dem behandelnden Neurologen eine Verschiebung des nächsten Therapiezyklus sinnvoll sein $[5,8,37,88-91]$.

\section{Impfempfehlungen - COVID-19 und MS}

Insgesamt wurden bisher 14 neue Impfstoffe zugelassen, die sich gegen SARSCoV-2 richten $[4,11,34,50,51,59$, $77,85,92]$. Innerhalb der EU wurden bereits 4 Impfstoffe ( 2 mRNA- und 2 nichtreplizierende Vektorvirusimpfstoffe) zugelassen. Für all diese 4 Impfstoffe liegen gute Wirksamkeitsdaten (67-95\%) vor [4, 59, 77, 85]. Darüber hinaus konnte in einer schottischen postapprovalen Studie eine 94 \%ige Reduktion der Krankenhausaufenthalte gezeigt werden, bei einem Studienkollektiv von mehrheitlich über 80-Jährigen [93]. Eine Folgestudie aus Schottland ergab für den BionTech/Pfizer- und AstraZenecaImpfstoff bereits 28 bis 34 Tage nach der ersten Impfung eine Reduktion der 
Tab. 1 Überblick über die wichtigsten neurologischen Manifestationen mit ihren einzelnen Diagnosen und vermuteten Pathomechanismen [15, 25, $45,49,52,56,61]$

\begin{tabular}{|c|c|c|}
\hline $\begin{array}{l}\text { Neurologische Manifes- } \\
\text { tation (COVID-19) }\end{array}$ & Beschriebene Krankheitsbilder & Pathomechanismus \\
\hline Enzephalopathie & Delir, Psychose, Vigilanzminderung & Multifaktoriell (Sepsis, Hypoxie, Immunhyperstimulation/CSS) \\
\hline $\begin{array}{l}\text { Entzündliche } \\
\text { ZNS-Syndrome }\end{array}$ & $\begin{array}{l}\text { Enzephalitis, ADEM (teils mit ICB, teils mit Nekrose), } \\
\text { transverse Myelitis }\end{array}$ & $\begin{array}{l}\text { Para-/postinfektiöser Mechanismus (z. B.: „molecular mimicry“, } \\
\text { „bystander activation“, „epitope spreading“) }\end{array}$ \\
\hline Schlaganfall & Ischämisch, Mikrohämorrhagien & $\begin{array}{l}\text { Begleitvaskulitis, prothrombotischer Status/Hyperkoagulabilität, } \\
\text { endotheliale Dysfunktion für Mikrohämorrhagien }\end{array}$ \\
\hline Periphere Beteiligung & GBS, Plexopathie & $\begin{array}{l}\text { Para-/postinfektiöser Mechanismus (z. B.: "molecular mimicry“, } \\
\text { „bystander activation“, „epitope spreading") }\end{array}$ \\
\hline $\begin{array}{l}\text { Postakutes } \\
\text { COVID-19-Syndrom ( } 38 \% \\
\text { neurologische Manifesta- } \\
\text { tionen) }\end{array}$ & $\begin{array}{l}\text { Fatigue, Kopfschmerzen, Dysautonomie, kognitive } \\
\text { Beeinträchtigung }\end{array}$ & $\begin{array}{l}\text { Immundysregulation, Inflammation, mikrovaskuläre Thrombosen, } \\
\text { iatrogene Wirkungen von Medikamenten }\end{array}$ \\
\hline Andere & $\begin{array}{l}\text { Hyposmie/Anosmie, Myelopathie (MR-negativ), } \\
\text { bilaterale Abduzensparese, nonkonvulsiver Status } \\
\text { epilepticus (NCSE), bakterieller Abszess }\end{array}$ & $\begin{array}{l}\text { Migration in das ZNS via N. olfactorius, bilaterale Abduzensparese } \\
\text { bei idiopathischer intrakranieller Hypertension, NCSE bei pädiatri- } \\
\text { schen Patienten mit bekannter Epilepsie }\end{array}$ \\
\hline
\end{tabular}

COVID-19-bedingten Spitalsaufenthalte um 88-91\% [75]. Ähnliche Ergebnisse liefert eine Studie an Mitarbeitern im Gesundheitswesen [22]. Dem folgend gibt es eine klare Empfehlung der WHO für die Anwendung bei $>65$-jährigen Personen, insbesondere den AstraZenecaImpfstoff betreffend (in der Zulassungsstudie nur 9,8\%>65 Jahre) [77, 94]. Auch das Sicherheitsprofil der in der EU zugelassenen Impfstoffe ist gut, insbesondere unter Berücksichtigung von bereits beinahe 900 Mio. verabreichten Impfstoffdosen weltweit (laut WHO bis zum 22.04.2021; [1, 86]).

In Zusammenhang damit muss jedoch angemerkt werden, dass bis zum 04.04.2021 insgesamt 169 Fälle von zerebraler Venenthrombose (CVT) und 53 Fälle von splanchnischer Venenthrombose an EudraVigilance nach AstraZeneca ( ${ }^{\circledR}$ Vaxzevria) gemeldet wurden, bei über $34 \mathrm{Mio}$. geimpften Menschen in der EU und Großbritannien [12, 48, 66, 68, 95]. Diese Daten ändern nichts an den Empfehlungen des Sicherheitsausschusses (PRAC) der EMA für den Impfstoff AstraZeneca $\left({ }^{\circledR}\right.$ Vaxzevria), dass der Nutzen dem Risiko klar überwiegt. So hat beispielsweise die STIKO in Deutschland die Empfehlung herausgegeben, dass Menschen unter 60 Jahren nicht mehr mit diesem Impfstoff geimpft werden sollen und Personen mit einer bereits erhaltenen Impfung als 2. Impfung einen mRNAImpfstoff im Sinne eines heterologen
Impfschemas erhalten sollen [96]. In einer Arbeit von Greinacher et al. wird die Bildung plättchenaktivierender Antikörper als möglicher Mechanismus für eine prothrombotische Thrombozytopenie nach der Impfung postuliert [20]. Auch im Zusammenhang mit dem Impfstoff von Johnson\&Johnson wurden 6 CVTs berichtet. Diesbezüglich gab es jedoch eine Sicherheitsüberprüfung durch die FDA (Food and Drug Administration) und das CDC (Centers for Disease Control and Prevention) und es wurde konsekutiv die Empfehlung zur Pausierung der Notzulassung wieder aufgehoben [97]. Nichtsdestotrotz können aktuell potenzielle Langezeitschäden nicht vollends ausgeschlossen werden, weswegen ein entsprechendes Surveillance-Programm notwendig ist. Des Weiteren wurden im Falle des AstraZeneca-Impfstoffs 3 Myelitisfälle berichtet, auch wenn diese von einem unabhängigen Expertenkomitee als unwahrscheinlich mit der Impfung im Zusammenhang stehend beurteilt wurden [77].

Darüber hinaus haben sich durch die rezente Zulassung des Johnson\&JohnsonImpfstoffs in der EU (11.03.2021), nach dem dieser bereits Ende Februar eine Notzulassung durch die FDA erhalten hatte, die Alternativen der Impfstoffe erweitert [85, 98]. Der Vorteil dieses Impfstoffs könnte, insbesondere im Kampf gegen die aufkommenden Virusmutationen sein, dass er nur einmalig verabreicht werden muss (Gefahr der
Resistenzbildung geringer; [47]). Die Wirksamkeit dieses Impfstoffes lag nach 14 Tagen bei $66 \%$ und nach 28 Tagen zwischen $57-72 \%$ und ist damit am ehesten vergleichbar mit dem AstraZenecaImpfstoff [99]. Bezüglich des russischen Sputnik-V-Impfstoffs wurden am 02.02.2021 die Phase-III-Studiendaten veröffentlicht, mit einer Wirksamkeit von 91,6\%. Das Sicherheitsprofil dieser beiden nichtreplizierenden Vektorvirusimpfstoffe ist, soweit dies aus den bisherigen Veröffentlichungen ersichtlich ist, ebenfalls gut $[34,85,99]$. Insgesamt sind alle bis dato in der EU zugelassenen SARS-CoV-2-Impfstoffe für MSPatienten zu empfehlen. Angesichts der 3 Myelitisfälle in den AstraZenecaImpfstoffstudien sowie der CVT-Fälle als auch der tendenziell höheren Wirksamkeit der mRNA-Impfstoffe, ist hier eine gewisse Überlegenheit der mRNAImpfstoffe gegeben. Dies entspricht auch den jeweiligen regionalen Empfehlungen [88-91]. Insbesondere den AstraZenecaImfpstoff betreffend müssen die Empfehlungen der STIKO berücksichtigt werden (nur bei $>60$-Jährigen).

Da es sich bei diesen 4 Impfstoffen um keine Lebendimpfstoffe handelt, besteht keine Gefahr einer Erkrankung, durch die Impfung. Gegebenenfalls kann es bei Patienten unter Immuntherapie zu einer reduzierten Immunantwort kommen $[32,76]$. Insbesondere im Falle einer zyklischen Immuntherapie (Ocrelizumab, Rituximab, Alemtuzumab, Cladribin), 
kann ein entsprechender Aufschub der nächsten Gabe sinnvoll sein, um möglichst eine protektive Immunantwort generieren zu können. Alle Impfungen sollten ausschließlich in Absprache mit dem behandelnden Neurologen und unter Berücksichtigung der einzuhaltenden Zeitintervalle erfolgen.

\section{Schlussfolgerungen}

COVID-19 ist eine Pandemie, die zu einem weltweiten Ausnahmezustand geführt hat, der viele Bereiche trifft, insbesondere das Gesundheitswesen. Seit Anbeginn der Pandemie haben sich beinahe 150 Mio. Menschen mit SARS-CoV-2 infiziert und sind über 3 Mio. Menschen an COVID-19 verstorben [86]. Innerhalb des letzten Jahres gab es enorme wissenschaftliche Fortschritte, sodass inzwischen weltweit 14 Impfstoffe gegen SARS$\mathrm{CoV}-2$ zugelassen werden konnten und beinahe 900 Mio. Impfdosen (laut WHO bis zum 22.04.2021) verabreicht werden konnten $[4,11,34,50,51,59,77,85,86$, 92]. Gerade im Zeitalter der immunmodulatorischen und immunsuppressiven Therapien sowie angesichts der Entwicklungen im Bereich der Impfstofftechnologien stehen Menschen mit Multipler Sklerose vor neuartigen Herausforderungen und Informationsbedarf. In diesem Zusammenhang ist es wichtig, zu betonen, dass Menschen mit MS kein generell erhöhtes Risiko haben, an COVID19 zu erkranken oder einen schwereren Verlauf zu erleiden [35, 71]. Vielmehr scheinen Komorbiditäten, Alter und Behinderungsgrad (EDSS) entscheidend zu sein $[10,29,30]$. Es wird daher allen MSPatienten empfohlen, ihre Immuntherapie wie bisher fortzuführen, immer in Absprache mit dem behandelnden Neurologen. Gerade bei zyklischen Therapien (Ocrelizumab, Rituximab, Alemtuzumab, Cladribin) kann eine mögliche Aufschiebung der nächsten Dosis sinnvoll sein [89]. Derzeit wird allen MS-Patienten das Impfen gegen SARS-CoV-2 entsprechend den regionalen Vorgaben empfohlen. Nur so kann der COVID-19Pandemie Einhalt geboten werden und ein Ende dieser weltweiten Gesundheitskrise erreicht werden.

\section{Korrespondenzadresse}

\section{Tobias Monschein}

Universitätsklinik für Neurologie, Medizinische Universität Wien

Waehringer Guertel 18-20, 1090 Wien,

Österreich

tobias.monschein@meduniwien.ac.at

\section{Prof. Hans-Peter Hartung, FRCP}

Klinik für Neurologie, Universitätsklinikum

Düsseldorf, Medizinische Fakultät, Heinrich-

Heine-Universität

Moorenstraße 5, 40225 Düsseldorf,

Deutschland

hans-peter.hartung@uni-duesseldorf.de

Funding. Open access funding provided by Medical University of Vienna.

\section{Einhaltung ethischer Richtlinien}

Interessenkonflikt. T. Monschein, T.Zrzavy, M. Löbermann, A. Winkelmann, T. Berger, P. Rommer, H.P. Hartung und U.K. Zettl geben an, dass kein Interessenkonflikt besteht.

Da es sich hier um einen Review-Artikel handelt, beinhaltet dieser Beitrag keine Studien an Menschen oder Tieren und ist somit im Einklang mit nationalem Recht sowie der Deklaration von Helsinki von 1975 (in der aktuellen, überarbeiteten Fassung).

Open Access. Dieser Artikel wird unter der Creative Commons Namensnennung 4.0 International Lizenz veröffentlicht, welche die Nutzung, Vervielfältigung, Bearbeitung, Verbreitung und Wiedergabe in jeglichem Medium und Format erlaubt, sofern Sie den/die ursprünglichen Autor(en) und die Quelle ordnungsgemäß nennen, einen Link zur Creative Commons Lizenz beifügen und angeben, ob Änderungen vorgenommen wurden.

Die in diesem Artikel enthaltenen Bilder und sonstiges Drittmaterial unterliegen ebenfalls der genannten Creative Commons Lizenz, sofern sich aus der Abbildungslegende nichts anderes ergibt. Sofern das betreffende Material nicht unter der genannten Creative Commons Lizenz steht und die betreffende Handlung nicht nach gesetzlichen Vorschriften erlaubt ist, ist für die oben aufgeführten Weiterverwendungen des $\mathrm{Ma}$ terials die Einwilligung des jeweiligen Rechteinhabers einzuholen.

Weitere Details zur Lizenz entnehmen Sie bitte der Lizenzinformation auf http://creativecommons.org/ licenses/by/4.0/deed.de.

\section{Literatur}

1. Achiron A, Dolev M, Menascu Set al (2021) COVID19 vaccination in patients with multiple sclerosis: What we have learnt by February 2021. Mult Scler J. https://doi.org/10.1177/13524585211003476

2. Achiron A, Mandel M, Dreyer-Alster S et al (2021) Humoral immune response to COVID-19 mRNA vaccine in patients with multiple sclerosis treated with high-efficacy disease-modifying therapies.
Ther Adv Neurol Disord 14:1-8. https://doi.org/10. 1177/17562864211012835

3. Alonso R, Silva B, Garcea O et al (2021) COVID19 in multiple sclerosis and neuromyelitis optica spectrum disorder patients in Latin America: COVID-19 in MS and NMOSD patients in LATAM. Mult Scler Relat Disord 51:102886. https://doi.org/ 10.1016/j.msard.2021.102886

4. Baden LR, El Sahly HM, Essink B et al (2021) Efficacy and safety of the mRNA-1273 SARS-coV-2 vaccine. N Engl J Med 384:403-416. https://doi.org/10. 1056/nejmoa2035389

5. Barun B, GabelićT, Adamecletal (2021) Influence of delaying ocrelizumab dosing in multiple sclerosis due to COVID-19 pandemics on clinical and laboratory effectiveness. Mult Scler Relat Disord. https://doi.org/10.1016/j.msard.2020.102704

6. Bergmann CC, Lane TE, Stohlman SA (2006) Coronavirus infection of the central nervous system: host-virus stand-off. Nat Rev Microbiol 4:121-132. https://doi.org/10.1038/nrmicro1343

7. Borriello G, Ianniello A (2020) COVID-19 occurring during Natalizumab treatment: a case report in a patient with extended interval dosing approach. Mult Scler Relat Disord 41:102165. https://doi.org/ 10.1016/j.msard.2020.102165

8. Bose G, Galetta K (2021) Reactivation of SARSCoV-2 after Rituximab in a Patient with Multiple Sclerosis. Multiple sclerosis and related disorders 52:102922. https://doi.org/10.1016/j.msard.2021. 102922

9. Browne $P$, Chandraratna D, Angood C et al (2014) Atlas of multiple sclerosis 2013: a growing global problem with widespread inequity. Neurology 83:1022-1024. https://doi.org/10.1212/WNL. 0000000000000768

10. Bsteh G, Bitschnau C, Hegen H et al (2020) Multiple sclerosis and COVID-19: how many are at risk? Eur J Neurol.https://doi.org/10.1111/ene.14555

11. ChiCTR2000039000 (2020) A Phase III clinical trial for inactivated novel coronavirus pneumonia (COVID-19) vaccine (Vero cells). http://www.who.int/trialsearch/Trial2.aspx? TrialID=ChiCTR2000039000, added to CENTRAL: 30 Nov 2020, 2020 Issue 11

12. Cines DB, Bussel JB (2021) E d i t o r i a I SARS-CoV-2 vaccine-induced immune thrombotic thrombocytopenia https://doi.org/10.1056/NEJM

13. Corman VM, Muth D, Niemeyer D, Drosten C (2018) Hosts and sources of endemic human Coronaviruses. Advances in Virus Research.https:// doi.org/10.1016/bs.aivir.2018.01.001

14. D'Amico F, Baumgart DC, Danese S, PeyrinBiroulet $L$ (2020) Diarrhea during COVID-19 infection: pathogenesis, epidemiology, prevention, and management. Clin Gastroenterol Hepatol 18:1663-1672. https://doi.org/10.1016/j.cgh. 2020.04.001

15. Egervari K, Thomas C, Lobrinus JA et al (2021) Neuropathology associated with SARS-CoV-2 infection. Lancet 397:276-277. https://doi.org/10. 1016/50140-6736(21)00095-7

16. Fajgenbaum DC, June CH (2020) Cytokine storm. N Engl J Med 383:2255-2273. https://doi.org/10. 1056/nejmra2026131

17. Galipeau Y, Greig M, Liu G et al (2020) Humoral responses and serological assays in SARS-CoV-2 infections. Front Immunol. https://doi.org/10. 3389/fimmu.2020.610688

18. Giacomelli A, Pezzati L, Conti F et al (2020) Self-reported olfactory and taste disorders in patients with severe acute respiratory coronavirus 2 infection: a cross-sectional study. Clin Infect Dis 71:889-890.https://doi.org/10.1093/cid/ciaa330 
19. Gorbalenya AE, Baker SC, Baric RS et al (2020) The species Severe acute respiratory syndrome-related coronavirus: classifying 2019-nCoV and naming it SARS-CoV-2. Nat Microbiol 5:536-544. https://doi. org/10.1038/s41564-020-0695-z

20. Greinacher A, Thiele T, Warkentin TE, Weisser K (2021) A prothrombotic thrombocytopenic disorder resembling heparin-induced thrombocytopenia following Coronavirus-19 vaccination. Res Sq Preprint. https://doi.org/10.21203/rs.3.rs362354/v1

21. Haji Abdolvahab M, Moradi-kalbolandi S, Zarei M et al (2021) Potential role of interferons in treating COVID-19 patients.Int Immunopharmacol.https:// doi.org/10.1016/j.intimp.2020.107171

22. Hall VJ, Foulkes S, Saei A et al (2021) Effectiveness of BNT162b2 mRNA vaccine against infection and COVID-19 vaccine coverage in healthcare workers in england, multicentre prospective cohort study (the SIREN study). SSRN Electron J. https://doi.org/ 10.2139/ssrn.3790399

23. Hauer L, Perneczky J, Sellner J (2020) A global view of comorbidity in multiple sclerosis: a systematic review with a focus on regional differences, methodology, and clinical implications. J Neurol. https://doi.org/10.1007/s00415-020-10107-y

24. Huang C, Wang Y, Li X et al (2020) Clinical features of patients infected with 2019 novel coronavirus in Wuhan, China. Lancet 395:497-506. https://doi. org/10.1016/S0140-6736(20)30183-5

25. ladecola C, Anrather J, Kamel H (2020) Effects of COVID-19 on the nervous system. Cell 183:16-27.e1.https://doi.org/10.1016/j.cell.2020. 08.028

26. Jack D, Damian D, Nolting A, Galazka A (2021) COVID-19 in patients with multiple sclerosis treated with cladribine tablets: an update. Mult Scler Relat Disord 51:102929. https://doi.org/10. 1016/j.msard.2021.102929

27. Jones SA, Hunter CA (2021) Is IL-6 a key cytokine target for therapy in COVID-19? Nat Rev Immunol. https://doi.org/10.1038/s41577-021-00553-8

28. Keddie S, Pakpoor J, Mousele C et al (2021) Epidemiological and cohort study finds no association between COVID-19 and Guillain-Barré syndrome. Brain 144:682-693. https://doi.org/10. 1093/brain/awaa433

29. Korsukewitz C, Reddel SW, Bar-Or A, Wiendl H (2020) Neurological immunotherapy in the era of COVID-19-looking for consensus in the literature. Nat Rev Neurol 16:493-505. https://doi.org/10. 1038/s41582-020-0385-8

30. Laroni A, Schiavetti I, Sormani MP, Uccelli A (2020) COVID-19in patients with multiple sclerosis undergoing disease-modifying treatments. Mult Scler J. https://doi.org/10.1177/1352458520971817

31. Larovere KL, Riggs BJ, Poussaint TY et al (2021) Neurologic involvement in children and adolescents hospitalized in the United States for COVID19 or multisystem inflammatory syndrome. JAMA Neurol.https://doi.org/10.1001/jamaneurol.2021. 0504

32. Löbermann M, Boršo D, Hilgendorf I et al (2012) Immunization in the adult immunocompromised host. Autoimmun Rev 11:212-218. https://doi. org/10.1016/j.autrev.2011.05.015

33. Loganathan SK, Schleicher K, Malik A et al (2020) Rare driver mutations in head and neck squamous cell carcinomas converge on NOTCH signaling. Science 367:1264-1269. https://doi.org/10.1126/ science.aax0902

34. Logunov DY, Dolzhikova IV, Shcheblyakov DV et al (2021) Safety and efficacy of an rAd26 and rAd5 vector-based heterologous prime- boost COVID-19 vaccine: an interim analysis of a randomised controlled phase 3 trial in Russia. Lancet 397:671-681. https://doi.org/10.1016/ S0140-6736(21)00234-8

35. Louapre C, Collongues N, Stankoff B et al (2020) Clinical characteristics and outcomes in patients with Coronavirus disease 2019 and multiple sclerosis. JAMA Neurol 77:1079-1088. https://doi org/10.1001/jamaneurol.2020.2581

36. Lu R, Zhao X, Li J et al (2020) Genomic characterisation and epidemiology of 2019 novel coronavirus: implications for virus origins and receptor binding. Lancet 395:565-574. https://doi.org/10.1016/ S0140-6736(20)30251-8

37. MaaroufA, Rico A, Boutiere Cetal (2020) Extending rituximab dosing intervals in patients with MS during the COVID-19 pandemic and beyond? Neurol Neuroimmunol Neuroinflamm. https://doi. org/10.1212/NXI.0000000000000825

38. Maghzi $\mathrm{AH}$, Houtchens MK, Preziosa $P$ et al (2020) COVID-19 in teriflunomide-treated patients with multiple sclerosis. J Neurol 267:2790-2796. https://doi.org/10.1007/s00415-020-09944-8

39. Magyari M, Sorensen PS (2020) Comorbidity in multiplesclerosis. Front Neurol. https://doi.org/10. 3389/fneur.2020.00851

40. Marrie RA, Elliott L, Marriott J et al (2015) Effect of comorbidity on mortality in multiple sclerosis. Neurology 85:240-247. https://doi.org/10.1212/ WNL.0000000000001718

41. Marrie RA, Horwitz RI (2010) Emerging effects of comorbidities on multiple sclerosis. Lancet $\mathrm{Neu}$ rol 9:820-828. https://doi.org/10.1016/S14744422(10)70135-6

42. Mehta P, McAuley DF, Brown M et al (2020) COVID-19: consider cytokine storm syndromes and immunosuppression. Lancet 395:1033-1034. https://doi.org/10.1016/S0140-6736(20)30628-0

43. Mehta P, Porter JC, Chambers RC et al (2020) B-cell depletion with rituximab in the COVID-19 pandemic: where do we stand? Lancet Rheumato 2:e589-e590. https://doi.org/10.1016/S26659913(20)30270-8

44. Mentis AFA, Dardiotis E, Grigoriadis N et al (2017) Viruses and multiple sclerosis: from mechanisms and pathways to translational research opportunities. Mol Neurobiol 54:3911-3923. https://doi.org/ 10.1007/s12035-017-0530-6

45. Modin D, Claggett B, Sindet-Pedersen Cet al (2020) Acute COVID-19 and the incidence of ischemic stroke and acute myocardial infarction. Circulation. https://doi.org/10.1161/CIRCULATIONAHA.120. 050809

46. Möhn N, Konen FF, Pul R et al (2020) Experience in multiple sclerosis patients with COVID-19 and disease-modifying therapies: a review of 873 published cases.JClin Med 9:4067.https://doi.org/ 10.3390/jcm9124067

47. Moore JP (2021) Approaches for optimal use of different COVID-19 vaccines: issues of viral variants and vaccine efficacy. J Am Med Assoc 325:1251-1252. https://doi.org/10.1001/jama. 2021.3465

48. Muir K-L, Kallam A, Koepsell SA et al (2021) Thrombotic Thrombocytopenia after Ad26.COV2.S Vaccination. N Engl J Med 384:1964-1965. https:// doi.org/10.1056/nejmc2105869

49. Nalbandian A, Sehgal K, Gupta A et al (2021) Postacute COVID-19 syndrome. Nat Med 27:601-615 https://doi.org/10.1038/s41591-021-01283-z

50. NCT04471519 (2020) Whole-Virion Inactivated SARS-CoV-2 Vaccine (BBV152) for COVID19 in Healthy Volunteers. https://clinicaltrials. gov/show/NCT04471519, available at https:// www.cochranelibrary.com/central/doi/10.1002 central/CN-02130159/full

51. NCT04527575 (2020) Study of the Safety, Reactogenicity and Immunogenicity of "EpiVacCorona" Vaccine for the Prevention of COVID-19. https:// clinicaltrials.gov/show/NCT04527575

52. Netland J, Meyerholz DK, Moore S et al (2008) Severe acute respiratory syndrome Coronavirus infection causes neuronal death in the absence of encephalitis in mice transgenic for human ACE2. JVirol. https://doi.org/10.1128/jvi.00737-08

53. Ogbe A, Kronsteiner B, Skelly DT et al (2021) T cell assays differentiate clinical and subclinical SARS-CoV-2 infections from cross-reactive antiviral responses. Nat Commun. https://doi.org/10.1038/ s41467-021-21856-3

54. Oksbjerg NR, Nielsen SD, Blinkenberg $M$ et al (2021) Anti-CD20 antibody therapy and risk of infection in patients with demyelinating diseases. Mult Scler Relat Disord. https://doi.org/10.1016/j. msard.2021.102988

55. Parrotta E, Kister I, Charvet L et al (2020) COVID19 outcomes in MS: Observational study of early experience from NYU Multiple Sclerosis Comprehensive Care Center. Neurol Neuroimmunol Neuroinflamm. https://doi.org/10.1212/NXI. 0000000000000835

56. Paterson RW, Brown RL, Benjamin L et al (2020) The emerging spectrum of COVID-19 neurology: clinical, radiological and laboratory findings. Brain 143:3104-3120. https://doi.org/10.1093/brain/ awaa240

57. Pawlitzki M, Zettl UK, Ruck T et al (2020) Merits and culprits of immunotherapies for neurological diseases in times of COVID-19. EBioMedicine. https://doi.org/10.1016/j.ebiom.2020.102822

58. Peter AE, Sandeep BV, Rao BG, Kalpana VL (2021) Calming the storm: natural immunosuppressants as adjuvants to target the Cytokine storm in COVID19. Front Pharmacol 11:1-22. https://doi.org/10. 3389/fphar.2020.583777

59. Polack FP, Thomas SJ, Kitchin N et al (2020) Safety and efficacy of the BNT162b2 mRNA Covid-19 vaccine. N Engl J Med 383:2603-2615. https://doi. org/10.1056/nejmoa2034577

60. Politi LS, Salsano E, Grimaldi M (2020) Magnetic resonance imaging alteration of the brain in a patient with coronavirus disease 2019 (COVID19) and anosmia. JAMA Neurol 77:1028-1029. https://doi.org/10.1001/jamaneurol.2020.2125

61. Reichard RR, Kashani KB, Boire NA et al (2020) Neuropathology of COVID-19: a spectrum of vascular and acute disseminated encephalomyelitis (ADEM)-like pathology. Acta Neuropathol 140:1-6. https://doi.org/10.1007/s00401-020-02166-2

62. Rostami Mansoor S, Ghasemi-Kasman M (2021) Impact of disease-modifying drugs on the severity of COVID-19 infection in multiple sclerosis patients. J Med Virol 93:1314-1319. https://doi. org/10.1002/jmv.26593

63. Rydyznski Moderbacher C, Ramirez SI, Dan JM et al (2020) Antigen-specific adaptive immunity to SARS-coV-2 in acute COVID-19 and associations with age and disease severity. Cell 183:996-1012.e19.https://doi.org/10.1016/j.cell. 2020.09 .038

64. Salama C, Han J, Yau L et al (2021) Tocilizumab in patients hospitalized with Covid-19 pneumonia. N Engl J Med 384:20-30. https://doi.org/10.1056/ nejmoa2030340

65. Schmaljohn A (2013) Protective antiviral antibodies that lack neutralizing activity: precedents and evolution of concepts. Curr HIV Res 11:345-353. https://doi.org/10.2174/1570162×113116660057 
66. Schultz NH, Sørvoll IH, Michelsen AE et al (2021) Thrombosis and thrombocytopenia afterChAdOx 1 nCoV-19 Vaccination. N Engl J Med. https://doi. org/10.1056/nejmoa2104882

67. Schultze JL, Aschenbrenner AC (2021) COVID19 and the human innate immune system. Cell 184:1671-1692. https://doi.org/10.1016/j.cell. 2021.02.029

68. Scully M, Singh D, Lown R et al (2021) Pathologic antibodies to platelet factor 4 after ChAdox $1 \mathrm{ncoV}$ 19 vaccination. N Engl J Med. https://doi.org/10. 1056/NEJMoa2105385

69. Sellner J, Rommer PS (2021) Multiple sclerosis and SARS-CoV-2 vaccination: considerations fo immune-depleting therapies. Vaccines 9:1-12. https://doi.org/10.3390/vaccines9020099

70. Sette A, Crotty S (2021) Adaptive immunity to SARS-CoV-2 and COVID-19. Cell 184:861-880. https://doi.org/10.1016/j.cell.2021.01.007

71. Sormani MP (2020) An Italian programme for COVID-19 infection in multiple sclerosis. Lancet Neurol 19:481-482. https://doi.org/10.1016/ S1474-4422(20)30147-2

72. Sormani MP, De Rossi N, Schiavetti I et al (2021) Disease-modifying therapies and Coronavirus disease 2019 severity in multiple sclerosis. Ann Neurol 89:780-789. https://doi.org/10.1002/ana. 26028

73. Thwaites RS, Uruchurtu ASS, Siggins MK et al (2021) Inflammatory profiles across the spectrum of disease reveal a distinct role for GM-CSF in severe COVID-19. Scilmmunol 6:1-17.https://doi.org/10. 1126/SCIIMMUNOL.ABG9873

74. Vabret N, Britton GJ, Gruber C et al (2020) Immunology of COVID-19: current state of the science. Immunity 52:910-941. https://doi.org/10. 1016/j.immuni.2020.05.002

75. Vasileiou E, Simpson CR, Shi T et al (2021) Interim findings from first-dose mass COVID19 vaccination roll-out and COVID-19 hospital admissions in Scotland: a national prospective cohort study. Lancet. https://doi.org/10.1016/ S0140-6736(21)00677-2

76. Velikova T, Georgiev T (2021) SARS-CoV-2 vaccines and autoimmune diseases amidst the COVID-19 crisis. Rheumatol Int 41:509-518. https://doi.org/ 10.1007/s00296-021-04792-9

77. Voysey M, Clemens SAC, Madhi SA et al (2021) Safety and efficacy of the ChAdOx $1 \mathrm{nCoV}-19$ vaccine (AZD1222) against SARS-CoV-2: an interim analysis of four randomised controlled trials in Brazil, South Africa, and the UK. Lancet397:99-111. https://doi.org/10.1016/S0140-6736(20)32661-1

78. Ware LB (2003) Severe acute respiratory syndrome In: Acute Respir. Distress Syndr, S 544-554

79. Wei WE, Li Z, Chiew CJ et al (2020) Presymptomatic transmission of SARS-coV-2-Singapore, january 23-march 16, 2020. MMWR Morb Mortal Wkly Rep 69:411-415.https://doi.org/10.15585/mmwr $\mathrm{mm} 6914 \mathrm{e}$

80. von Weyhern $\mathrm{CH}$, Kaufmann I, Neff F, Kremer M (2020) Early evidence of pronounced brain involvement in fatal COVID-19 outcomes. Lancet 395:e109. https://doi.org/10.1016/S01406736(20)31282-4

81. Winkelmann A, Loebermann $M$, Reisinger EC et al (2016) Disease-modifying therapies and infectious risks in multiple sclerosis. Nat Rev Neuro 12:217-233. https://doi.org/10.1038/nrneurol. 2016.21

82. Wouk J, Rechenchoski DZ, Rodrigues BCD et al (2021) Viral infections and their relationship to neurological disorders. Arch Virol 166:733-753. https://doi.org/10.1007/s00705-021-04959-6
83. Zhang H, Kang Z, Gong Het al (2020) The digestive system is a potential route of 2019-nCov infection: a bioinformatics analysis based on single-cell transcriptomes. bioRxiv. https://doi.org/10.1101/ 2020.01.30.927806

84. Zhou F, Yu T, Du R et al (2020) Clinical course and risk factors for mortality of adult inpatients with COVID-19 in Wuhan, China: a retrospective cohort study. Lancet 395:1054-1062. https://doi.org/10. 1016/S0140-6736(20)30566-3

85. https://www.ema.europa.eu/en/news/emarecommends-covid-19-vaccine-janssenauthorisation-eu.Zugegriffen: 11. März 2021

86. https://covid19.who.int/. Zugegriffen: 24. Apr. 2021

87. https://www.who.int/news-room/commentaries/ detail/transmission-of-sars-cov-2-implicationsfor-infection-prevention-precautions. Zugegriffen:01. März 2021

88. https://www.oegn.at/covid-19/covid19-undmultiple-sklerose-ms/. Zugegriffen: 01. März 2021

89. https://www.msif.org/news/2020/02/10/thecoronavirus-and-ms-what-you-need-to-know/. Zugegriffen:01.Apr. 2021

90. https://www.mssociety.org.uk/what-we-do/ news/ms-society-medical-advisers-releaseconsensus-statement-covid-19-vaccines. Zugegriffen: 12. März 2021

91. https://www.nationalmssociety.org/coronaviruscovid-19-information/multiple-sclerosisand-coronavirus/covid-19-vaccine-guidance/ Timing-MS-Medications-with-COVID-19-mRNAVaccines. Zugegriffen:01. Apr. 2021

92. https://covid19.trackvaccines.org/vaccines/. Zugegriffen:20. Apr. 2021

93. https://www.astrazeneca.com/media-centre/ articles/2021/first-real-world-study-of-covid19-vaccine-astrazeneca-demonstrates-94reduction-in-hospitalisations.html. Zugegriffen: 23. Feb. 2021

94. https://www.who.int/publications/i/item/WHO2019-nCoV-vaccines-SAGE recommendationAZD1222-2021.1.Zugegriffen:21. Apr. 2021

95. https://www.ema.europa.eu/en/news/astrazenecascovid-19-vaccine-ema-finds-possible-link-veryrare-cases-unusual-blood-clots-low-blood. Zugegriffen:07.Apr. 2021

96. https://www.rki.de/DE/Content/Kommissionen/ STIKO/Empfehlungen/Stellungnahme-Impfabstand. html\#: :text=Die\%20STIKO\%20empfiehlt\%2C \%20die\%20Impfung, dem\%20AstraZeneca \%2DImpfstoff\%20zu\%20verabreichen. Zugegriffen: 14.Apr. 2021

97. https://www.fda.gov/news-events/pressannouncements/fda-and-cdc-lift-recommendedpause-johnson-johnson-janssen-covid-19vaccine-use-following-thorough. Zugegriffen: 23 . Apr. 2021

98. https://www.fda.gov/emergency-preparednessand-response/coronavirus-disease-2019-covid19/janssen-covid-19-vaccine. Zugegriffen: 23 Apr. 2021

99. https://www.janssen.com/germany/covid-19impfstoffkandidat-zwischenergebnisse-derphase-3-studie.Zugegriffen:23. Apr. 2021

\section{Neuro Update Topaktuell und praxisnah}

04. und 05. März aus Mainz 18. und 19. März aus Berlin

Umfangreiches Update-Wissen so flexibel wie noch nie: Das Neurologie-UpdateSeminar findet als Hybridveranstaltung statt. Bilden Sie sich vor Ort mit Kolleginnen und Kollegen oder bequem von zuhause aus fort - die Zertifizierung ist für beide Formate beantragt.

Das Update-Konzept: Die wichtigsten Neuerungen des vergangenen Jahres aus der Neurologie werden unter der wissenschaftlichen Leitung von Prof. Dr. Gerhard Hamann (Günzburg), Prof. Dr. Marianne Dieterich (München), Prof. Dr. Volker Limmroth (Köln) und Prof. Dr. Heinz Reichmann (Dresden) kritisch selektiert, analysiert und zusammengefasst. Die Relevanz für Ihren Klinik- und Praxisalltag steht dabei im Vordergrund und der ausführlichen Diskussion über den Livechat mit den Referierenden wird viel Raum gegeben.

In diesem Jahr werden neben den Kerngebieten der Neurologie die Themen »Periphere Neurologie/Polyneuropathien«, »Round Table COVID-19« und »Neurogenetik« vorgestellt.

Zum Gesamtpaket der Teilnahme gehören neben den umfangreichen Seminarunterlagen der Download aller Vortragspräsentationen und die Vorträge im Nachgang als Video-on-Demand auf der Videoplattform »streamed-up.com«.

Weitere Informationen zu Programm Referierenden und zur Anmeldung: www.neuro-update.com

Veranstalter: med update $\mathrm{GmbH}$ Hagenauer Straße 53 65203 Wiesbaden 